\title{
Recent Developments in the Diagnosis and Treatment of Ocular Toxoplasmosis
}

\author{
Cem Ozgonul Cagri Giray Besirli \\ Department of Ophthalmology and Visual Sciences, University of Michigan Medical School, Ann Arbor, Mich., USA
}

\section{Key Words}

Uveitis - Retinochoroiditis · Parasitic eye infection .

Clindamycin · Pyrimethamine $\cdot$ Sulfadiazine

\begin{abstract}
Ocular toxoplasmosis, a chorioretinal infection with Toxoplasma gondii, is the most common etiology of posterior uveitis in many countries. Accurate diagnosis depends heavily on the characteristic clinical features of this disease, but atypical presentations, especially in immunocompromised patients, may create diagnostic challenges and lead to misdiagnosis and inappropriate treatment. Molecular biology techniques to diagnose ocular toxoplasmosis have been available for many years and are now accessible as standard laboratory tests in many countries. Aqueous humor or vitreous evaluation to detect parasite DNA by polymerase chain reaction or specific antibody may provide definitive evidence for rapid diagnosis. Oral pyrimethamine and sulfadiazine plus systemic corticosteroids are an effective therapy for ocular toxoplasmosis. Recent data supports the use of other treatment approaches, including intravitreal antibiotics. The aim of the present review is to discuss briefly the new diagnostic tools and treatment options for ocular toxoplasmosis.

(c) 2016 S. Karger AG, Basel
\end{abstract}

\section{Introduction}

Toxoplasma gondii is a ubiquitous intracellular parasite that infects both humans and warm-blooded animals. T. gondii is a leading infectious cause of posterior uveitis worldwide [1]. In high T. gondii endemic regions of the USA and Europe, ocular toxoplasmosis is the most frequent cause of posterior uveitis, presenting with a unilateral chorioretinal lesion associated with vitritis $[2,3]$. Although ocular toxoplasmosis in adult life was presumed to be the recurrence of the congenitally acquired infection, more recent reports indicate that acquired infections may account for a larger portion of ocular involvement than congenital toxoplasmosis $[4,5]$.

T. gondii, a member of the phylum Apicomplexa, has a polar apical complex that mediates attachment to the host cell membrane [6]. T. gondii exists in 3 infectious forms including sporozoites, which are contained within oocysts, tachyzoites and bradyzoites, which reside in tissue cysts. Oocysts are produced only in cat intestines and require sexual reproduction. Sporulated oocysts measure approximately $10 \mu \mathrm{m}$ and contain 2 sporocysts, and each sporocyst contains 4 sporozoites surrounded by a cell wall. These forms then spread out with defecation and become infectious in 1-5 days by sporulation. Tachyzo-

\section{KARGER}

(c) 2016 S. Karger AG, Basel

E-Mail karger@karger.com

www.karger.com/ore
Cagri Giray Besirli, MD, PhD

Department of Ophthalmology and Visual Sciences

University of Michigan Medical School

Ann Arbor, MI 48105 (USA)

E-Mail cbesirli@umich.edu 


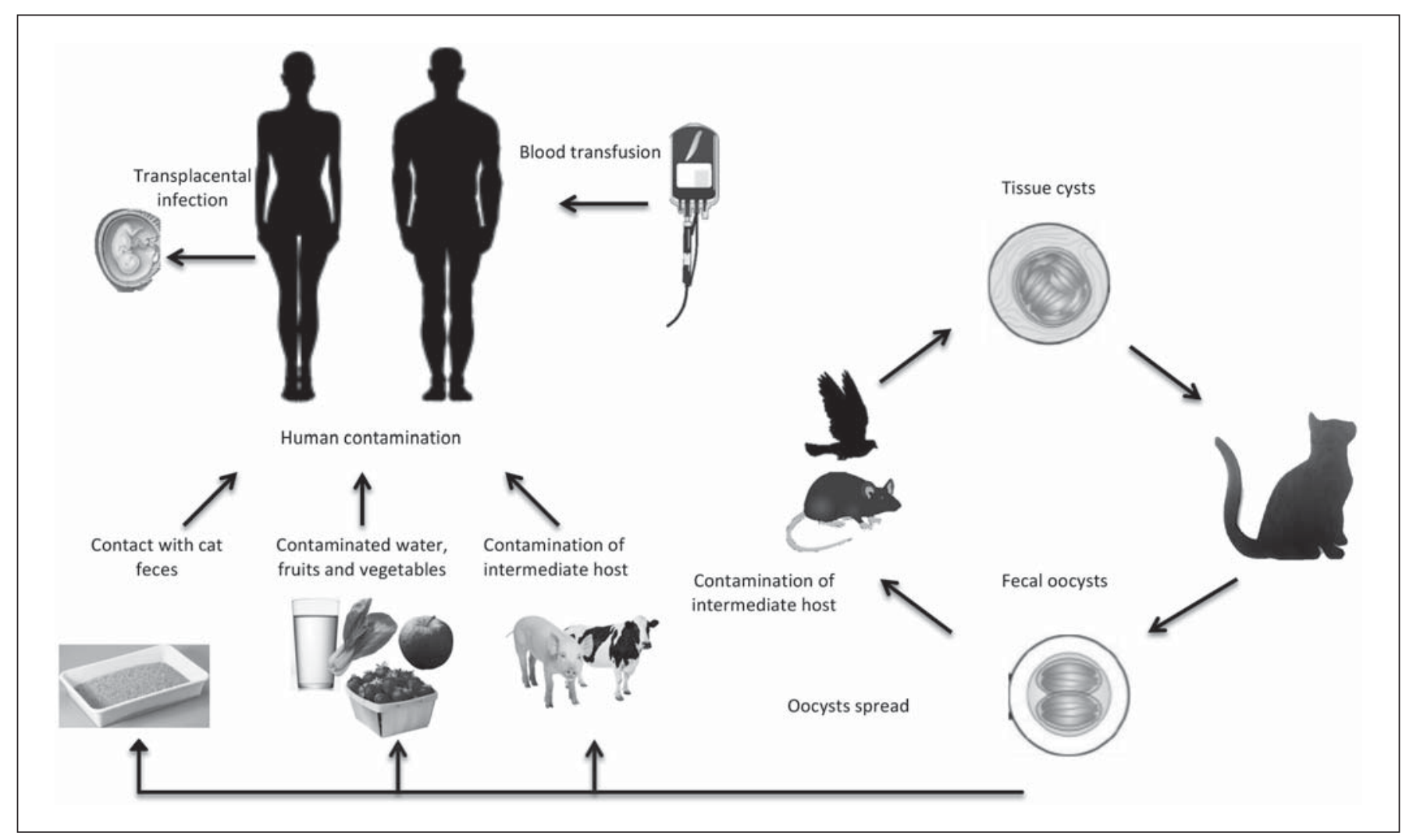

Fig. 1. Life cycle of T. gondii and the routes of infection.

ites are in crescentic form, which has the apical complex in one end, and are about $6 \times 2 \mu \mathrm{m}$ in size. This is the fastest replicating form and responsible for systemic dissemination and active tissue infection in intermediate hosts. Tachyzoites can enter almost any type of host cell and multiply until the host cell is filled with parasites. Lysis of the host cell results in tachyzoite release followed by reentry into a new host cell. As a result of this cycle, multifocal tissue necrosis may occur. The host usually limits this phase of infection, and the parasite then enters the dormant form, named bradyzoites. This form of parasite is characteristic for chronic infection, and bradyzoites are isolated in tissue cysts. Cysts may be up to $60 \mu \mathrm{m}$ in diameter and contain hundreds of bradyzoites. These cysts usually cause no host reaction and may remain throughout the life of the host.

Visual symptoms during acute toxoplasma retinochoroiditis are typically secondary to vitritis or less frequently from the involvement of the macula or optic nerve. Vision loss may become permanent due to formation of a macular scar or optic atrophy, and up to $24 \%$ of patients may have 20/200 vision or less in at least one eye [7, 8]. A toxoplasmosis scar can be associated with severe visual field loss when it occurs close to the optic disk [9].

Cats are the definitive hosts for T. gondii, and humans and other mammals act as intermediate hosts. The transmission occurs by many routes, including ingestion of raw or undercooked meat infected with tissue cysts, ingestion of food and water contaminated with oocysts, ingestion of eggs and milk contaminated with tachyzoites, blood transfusion, organ transplantation or transplacental transmission (fig. 1) [10].

Accurate diagnosis depends heavily on the characteristic clinical features of this disease, but atypical presentations, especially in immunocompromised patients, may create diagnostic challenges and lead to misdiagnosis and inappropriate treatment. Oral pyrimethamine and sulfadiazine plus systemic corticosteroids are an effective therapy for ocular toxoplasmosis. The purpose of this review is to present new developments on diagnostic methods and treatment options in patients with ocular toxoplasmosis. 


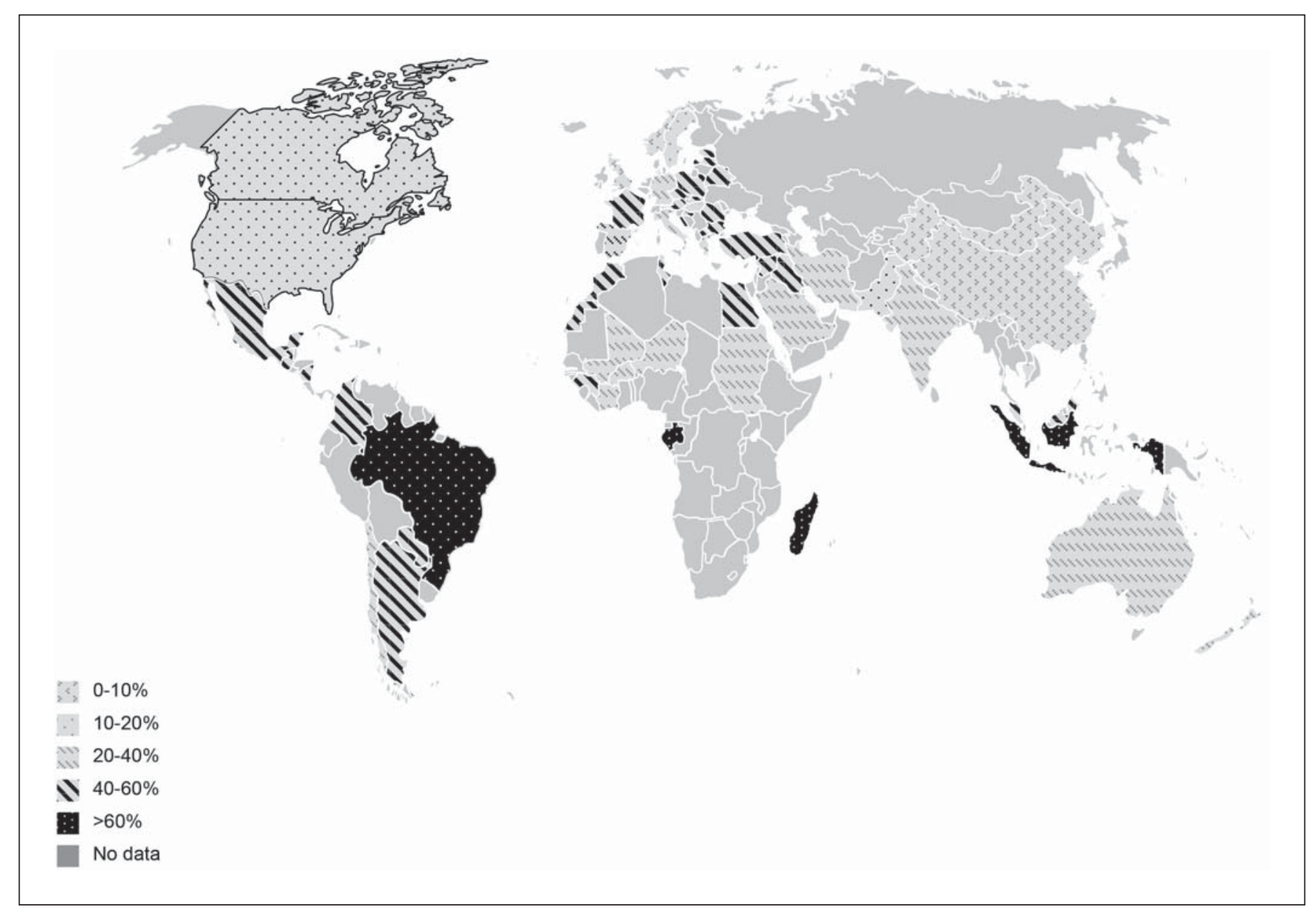

Fig. 2. Global seroprevalence of T. gondii.

\section{Epidemiology}

T. gondii is a common parasite that infects almost all species of mammals including humans. Approximately $25-30 \%$ of the human population is infected with T. gondii [11]. However, seroprevalence varies widely, from 10 to $80 \%$ between different geographic areas and countries and even within countries. Reports with low seroprevalence are from Southeast Asia, North America and Northern Europe with 10-30\% [12]. Prevalences between 30 and $50 \%$ have been reported for Central and Southern Europe, whereas high seroprevalences are observed in Latin America and in tropical African countries [13]. The most probable explanation for these different seropositivity rates is the prevalence of $T$. gondii cysts and oocysts in the environment (fig. 2) [14].
Most patients present with uveitis secondary to ocular toxoplasmosis in their second to fourth decade of life. Disease severity is typically higher in older patients $[15$, 16]. In a study by Nguyen et al. [17], toxoplasmosis was the most common etiology of uveitis in patients referred to a tertiary center and had a prevalence of $14 \%$ among all other etiologies. A larger study in Europe showed a lower incidence in a population of 3,080 patients with uveitis, where $T$. gondii infection was the underlying etiology in $2.8 \%$ of cases [18]. In this study, recurrent ocular disease occurred with a high rate ( $79 \%$ of cases), and the time lapse between recurrences shortens with ongoing disease [18]. A survey of 1,916 patients from Europe found ocular toxoplasmosis to be the most frequent diagnosis in patients with posterior uveitis and the cause of $4.2 \%$ of uveitis cases [19]. Multiple studies from different regions of the globe have identified ocular toxoplasmosis as the 
most common form of posterior uveitis [20]. In some populations, ocular toxoplasmosis is the leading cause of uveitis [20]. In North Africa, ocular toxoplasmosis is reported to be the second most common cause of uveitis $(10.8 \%)$ and the most common etiology of posterior uveitis (38.3\%) [21]. Similarly, a recent survey of pediatric patients with uveitis treated at one of three tertiary referral clinics across the USA identified ocular toxoplasmosis as the most common form of posterior uveitis in children [22]. Ocular toxoplasmosis is likely underdiagnosed in many countries with endemic $T$. gondii infection. Six percent of healthy subjects who underwent ocular examination had retinal scars attributed to ocular toxoplasmosis in Colombia [23]. One retrospective chart review in India identified ocular toxoplasmosis as the most common cause of posterior uveitis, accounting for a total of $12 \%$ of cases [24].

Ocular toxoplasmosis has long been regarded as a disease that was mainly caused by congenital infection, while symptomatic ocular infection acquired after birth was considered rare. This paradigm was challenged by a study from Brazil, which demonstrated that postnatal infection and ocular manifestations of toxoplasmosis were more common than congenital infection [25]. This finding was confirmed by other groups and likely applies to most populations exposed to T. gondii [5]. The incidence of congenital infection ranges from $1 / 770$ to $1 / 10,000$ and largely depends on the region of the world [26-28]. In neonates with congenital toxoplasmosis, the incidence of retinochoroiditis varies in different studies but may be as high as $80 \%$ [28]. In pediatric cases, ocular disease is the most common manifestation of congenital toxoplasmosis, with $95 \%$ of patients showing signs of chorioretinitis in the presence of systemic findings, and occurs in the absence of systemic involvement in $26 \%$ of children [ 29 , 30].

The majority of ocular toxoplasmosis is acquired orally, either by consuming or handling raw meat containing tissue cysts, or by drinking water contaminated with oocysts shed by cats [10]. Less commonly, the tachyzoite passes vertically from mother to fetus as seen in congenital toxoplasmosis. Pork, chicken and lamb are more likely sources of $T$. gondii infection than beef although in theory, ingestion of contaminated meat from any warmblooded animal can transmit toxoplasmosis [31]. Water reservoirs that become contaminated by feces of infected cats may deliver $T$. gondii oocysts to a large population in a short time interval [32]. This type of transmission leads to extensive outbreaks of toxoplasmosis as described in various parts of the developing world. The geographic distribution of human infections largely matches the distribution of municipal water reservoirs in proven T. gondii outbreaks [33].

\section{Clinical Features}

Ocular toxoplasmosis often presents with classic ophthalmic findings, and the diagnosis is reached by clinical examination without any laboratory confirmation of $T$. gondii infection [34]. Seropositivity for T. gondii infection indicates previous systemic exposure to the parasite, though this finding is not sufficient to confirm the diagnosis of ocular toxoplasmosis. Visual impairment may be secondary to a macular lesion, while lesions located at the peripheral retina often lead to vision loss secondary to severe vitreous inflammation $[35,36]$. Optic nerve involvement is less common but may induce severe visual field defects as well as loss of color vision. Active lesions are associated with symptomatic vitreous inflammation, leading to blurry vision as the presenting symptom of ocular toxoplasmosis. Scotomas are directly related to the size and location of retinochoroidal scars during the inactive stage of the parasite.

Classical ocular manifestation of toxoplasmosis is a nidus of fluffy white, focal necrotizing retinitis or retinochoroiditis adjacent to a variably pigmented chorioretinal scar (fig. 3). Often the active lesion is obscured by severe vitritis producing the classic 'headlight in the fog' sign [37]. The severity of anterior uveitis may range from minimal reaction to an intense inflammation, masking the posterior segment involvement. Anterior uveitis may be either granulomatous or nongranulomatous inflammation. In children with congenital toxoplasmosis, cataract may be associated with retinochoroiditis and may follow severe iridocyclitis [36]. Other common clinical signs of ocular toxoplasmosis include satellite lesion (adjacent to an inactive retinochoroidal scar as seen in fig. 4), retinochoroidal scar, focal or widespread vasculitis, and inflammatory ocular hypertension syndrome [38]. Atypical findings include multifocal retinochoroiditis, low-grade or absent vitreal infiltration, an active lesion more than 2 disk diameters without an associated retinochoroidal scar, absence of a retinochoroidal scar, bilaterality, optic disk involvement, choroiditis without retinitis, hemorrhagic vasculitis, serous retinal detachment, and retinal neovascularization [38].

Spectral-domain optical coherence tomography (SDOCT) imaging is an important diagnostic tool to identify the morphological features of the vitreoretinal changes in
Ozgonul/Besirli 


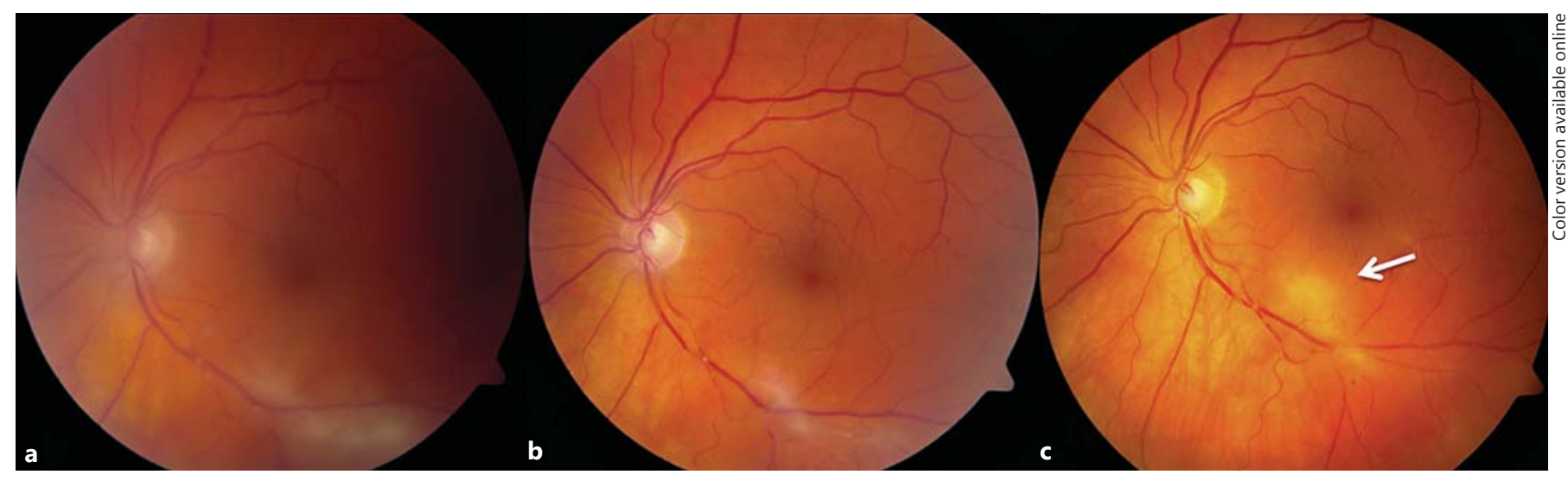

Fig. 3. a Fundus photograph of a 63-year-old male patient with active ocular toxoplasmosis chorioretinitis with local vitritis along the inferior vascular arcade. $\mathbf{b}$ Resolved vitritis and resolving lesion 2 weeks after starting dual therapy with trimethoprim-sulfa- methoxazole and prednisone. c Fibrosis and vitreoretinal adhesion with new focus of active retinitis closer to the fovea (white arrow). After the treatment of the new focus, trimethoprim-sulfamethoxazole prophylaxis continued for this patient.
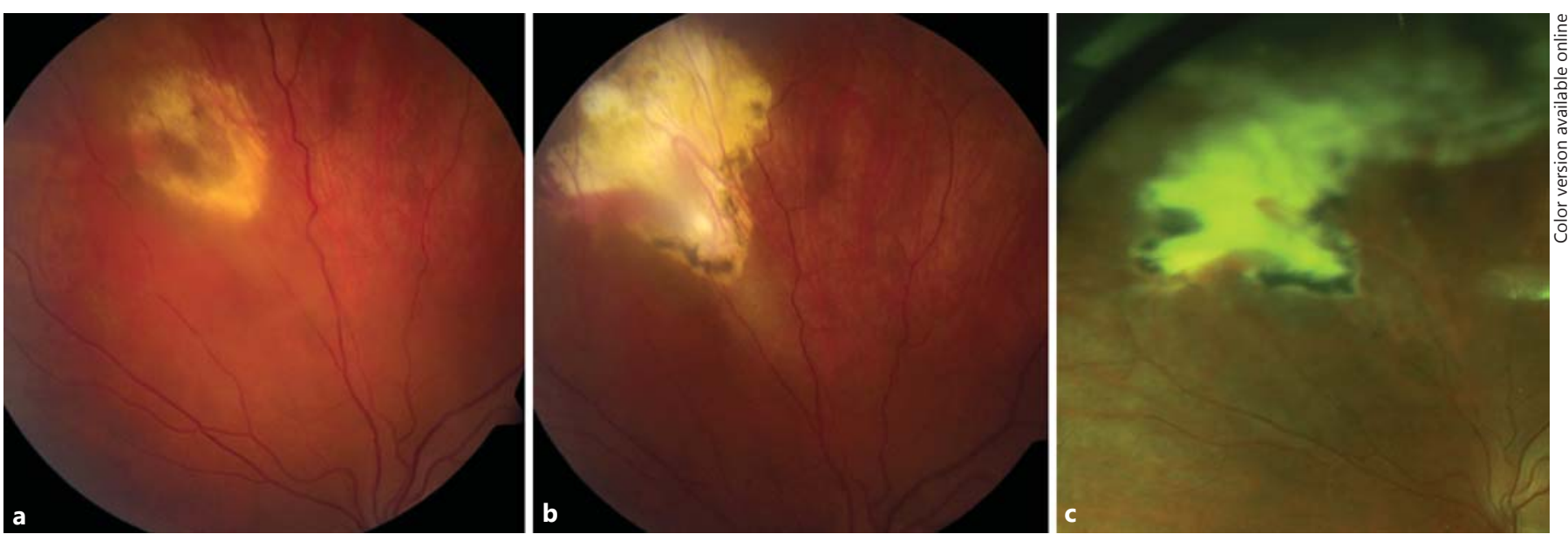

Fig. 4. a Primary manifestation of a peripheral retinochoroidal lesion in a 53-year-old immunocompetent male patient diagnosed with ocular toxoplasmosis by clinical appearance. b Active focal

ocular toxoplasmosis [39-41]. The stage of the disease is determinant for the SD-OCT findings of chorioretinal lesions. Goldenberg et al. [40] studied the vitreoretinal changes of ocular toxoplasmosis, during the acute phase, treatment phase, and after resolution, using SD-OCT. In the acute phase, disruption, thickening, and hyperreflectivity of the neurosensory retina with photoreceptor interruption and retinal pigment epithelial elevation were found. During follow-up, neurosensory retinal layer thinning and disorganization, photoreceptor interruption,

Ocular Toxoplasmosis retinitis adjacent to the old scarred lesion in the same patient. c Recurrent manifestation was observed within 5 years presenting with 'clustering' appearance. and retinal pigment epithelial elevation and/or atrophy were demonstrated. Multiple hyperreflective dots in the vitreous cavity, compatible with posterior vitritis and vitreous cells, and posterior hyaloid thickening with partial detachment were demonstrated in the acute phase. With improvement of disease, hyperreflective dots become smaller, and eventually resolve. The posterior hyaloid may thicken and detach during follow-up. An epiretinal membrane may be found over active as well as scarred lesions (fig. 5). 
Fig. 5. SD-OCT of a Toxoplasma chorioretinits lesion. a Vertical SD-OCT through the retinal lesion shows retinal thickening with full-thickness retinal hyperreflectivity (white arrow) extending down to the level of the retinal pigment epithelium and Bruch's membrane. Hyperreflective spots in the vitreous (white arrowhead) in the acute phase demonstrate posterior vitreous cell. b Thickening of the posterior hyaloid (white arrow) over the scar and epiretinal membrane formation (white arrowhead) in the chronic phase. c Thickened posterior hyaloid face and a posterior vitreous detachment (white arrowhead) over the lesion in the chronic phase.
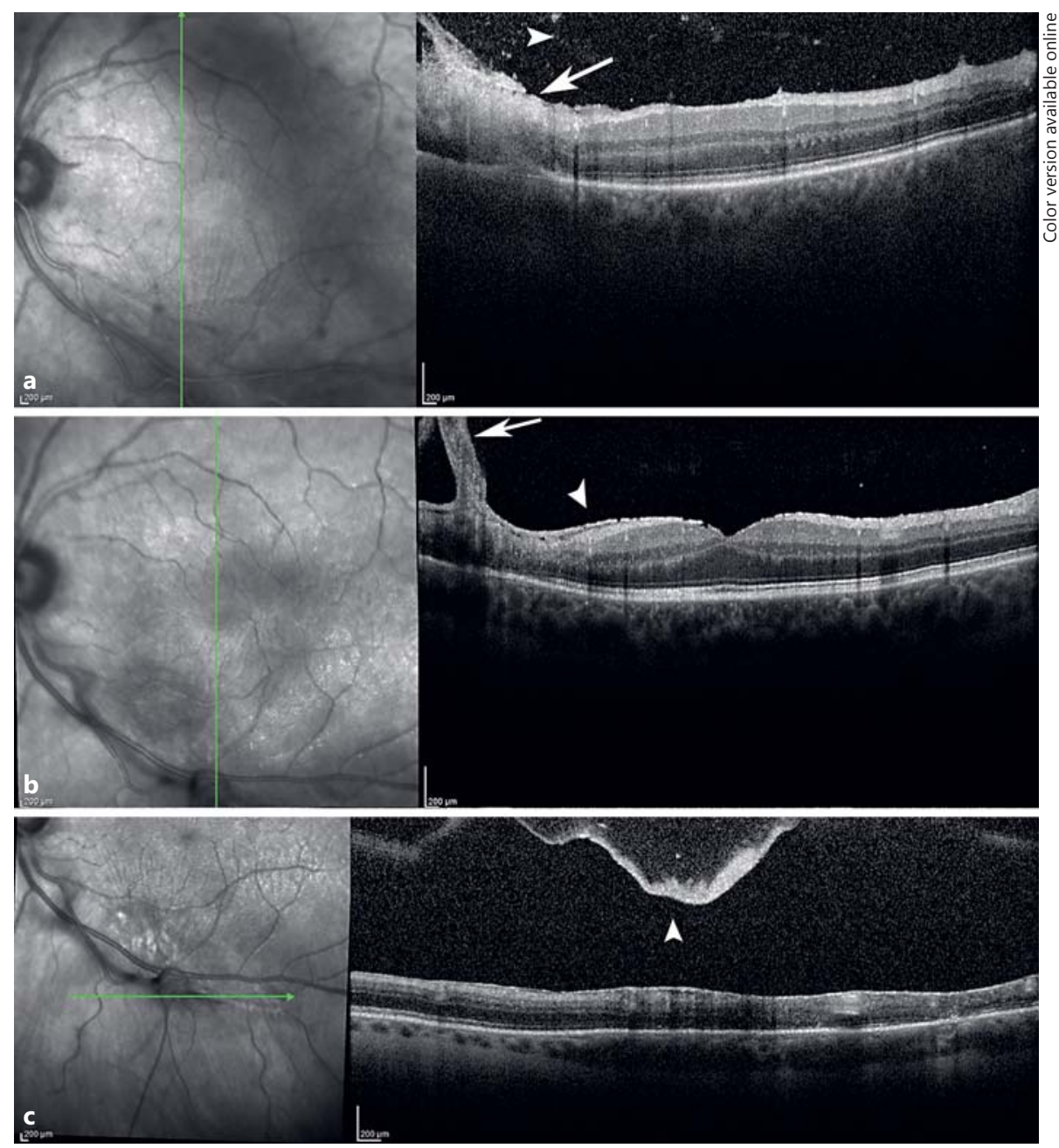

\section{Diagnosis}

The diagnosis of ocular toxoplasmosis is usually evident based on typical clinical presentation. When the clinical diagnosis cannot be made definitely by a funduscopic examination, serological tests including serum anti-Toxoplasma titers of IgM and IgG may be needed to support the diagnosis. T. gondii antibody titers in ocular fluids or polymerase chain reaction (PCR) of aqueous and vitreous samples are other newer tools with high sensitivity and specificity to confirm the diagnosis $[42,43]$.

Most diagnostic laboratories are only capable of measuring IgG and IgM antibody levels using enzyme-linked immunosorbent assay (ELISA) or immunofluorescent antibody commercial kits. ELISA has an advantage over immunofluorescent antibody testing because it permits automation for simultaneous testing of large numbers of samples and the results are objective [44]. The SabinFeldman dye test, the classic gold standard serology test, uses live T. gondii tachyzoites to detect IgG antibodies [44]. Despite its high sensitivity and specificity, this test is not frequently performed, owing to the risk for laboratory-acquired infections, and is available in very few reference laboratories in North America.

Serum IgM and IgG antibodies to T. gondii develop within 1-2 weeks after infection [45]. Patients suspected of acute toxoplasmosis may initially be analyzed for IgG serology primarily, and if the result is positive for IgG, IgM antibody levels may be measured. Nonreactive IgG rules out the toxoplasmosis diagnosis in an immunocompetent patient [10]. IgM levels rise within the first week and become undetectable after 6-9 months. Elevated levels of antibodies alone should not be considered as an evidence of recent infection, nor should low serum IgG 
levels be considered as inactive disease. If the laboratory testing is unequivocal, levels of serological tests should be repeated in 15-21 days [44].

Asymptomatic patients with IgG reactivity alone may have latent infection with a history of primary exposure. This serological pattern is most important for immunosuppressed patients, including HIV infection and transplant recipients, and defines the risk for reactivation of disease [10]. In patients with reactivation disease, IgM and IgG response may not be seen. In immunocompromised patients with seronegativity but strong clinical evidence, further tests to exclude Toxoplasma infection should be performed. These include IgG antibody testing or T. gondii PCR of the vitreous and aqueous humor.

Serology is also used to assess the risk of transplacental transmission. IgG serology is performed in women considering pregnancy routinely in countries with endemic toxoplasmosis [46]. Elevated levels of IgG before pregnancy in immunocompetent women indicate a low risk for transplacental transmission. Those with undetectable IgG levels are advised to avoid undercooked meat consumption or cat feces. Negative serology of IgM excludes acute infection in the last 6 months; if it is positive, it may persist up to 2 years after exposure to T. gondii. The IgG avidity test provides information about the time of exposure if serologies of IgG and IgM are reactive. An IgG avidity test resulting in high-avidity IgG antibodies in sera of patients in the first trimester indicates that the infection was acquired before conception, because high-avidity IgG antibodies take 3-4 months to appear [47]. Low-avidity IgG antibodies should not be used to confirm the diagnosis of recent infection, due to persistence of these antibodies for many months after the acute infection $[10,46]$.

Detection of Toxoplasma-specific antibodies or DNA of the parasite in ocular specimens is the main basis of the diagnosis [11]. Intraocular antibody production is established by the Goldmann-Witmer coefficient (GWC), which compares the Toxoplasma-specific antibodies in ocular fluids and in serum [48]. Although a ratio $>1$ should indicate intraocular antibody production, this may also occur in healthy controls, and therefore a ratio of at least 3 is often used to confirm diagnosis [49].

The contribution of PCR to help with the diagnosis is more controversial. In immunocompetent patients with the clinical diagnosis of ocular toxoplasmosis, DNA of $T$. gondii could be amplified by PCR techniques only in 30$40 \%$ cases $[50,51]$. However, in immunocompromised individuals, T. gondii DNA was amplified in $75 \%$ of the clinically diagnosed patients [51]. Montoya et al. [52] reported that the diagnostic value of PCR in intraocular specimens for T. gondii chorioretinitis was $67 \%$. The sensitivity of PCR in patients meeting clinical diagnostic criteria for toxoplasmic chorioretinitis was lower in other studies, ranging from 27 to $36 \%[49,52,53]$. Despite low sensitivity, the specificity of PCR is $100 \%$ [54]. PCR is a highly sensitive method of detecting nucleic acids, but no current standards are available to diagnose ocular toxoplasmosis, as reported by Garweg et al. [51], and this likely accounts for the wide range of PCR sensitivity in published reports. To improve the sensitivity of PCR, investigators are analyzing different DNA targets in patients with ocular toxoplasmosis. These studies are focused especially on Toxoplasma $\mathrm{B} 1$ gene, which is a promising genomic fragment, owing to the higher number of repeats and highly conserved DNA sequence $[55,56]$. The sensitivity of PCR also depends on the immune status of the patient. When the clinical symptoms first manifest in immunocompetent patients, the intraocular inflammatory response reduces the parasitic burden in the aqueous humor and vitreous, thus decreasing the amount of target DNA for PCR amplification.

Rothova et al. [43] compared the efficiency of PCR to GWC in the aqueous humor of patients with toxoplasmic chorioretinitis. Their results showed that GWC is a significantly more sensitive test. PCR was negative in $84 \%$ of toxoplasmic chorioretinitis patients, in contrast to $7 \%$ for GWC. Although PCR currently has low sensitivity, Fekkar et al. [50] reported that combining GWC with PCR significantly improved the diagnostic sensitivity from 81 to $93 \%$. The sensitivity and the specificity of intraocular antibody detection have been reported to be 63 and $89 \%$ by others, and adding PCR as an additional laboratory test may be of value in some cases [54]. In addition, GWC typically is more sensitive in immunocompetent patients, as the ocular antibody production is often unpredictable in immunocompromised patients. Rothova et al. [43] reported GWC sensitivity of $57 \%$ in immunocompromised patients, whereas the sensitivity was $93 \%$ in immunocompetent patients. Table 1 summarizes the diagnostic tests based on immune status of the patients.

To improve the sensitivity of PCR, Sugita et al. [56] established a 2-step PCR protocol as a novel PCR technique for the diagnosis of ocular toxoplasmosis. In the first step, this technique uses a qualitative multiplex PCR approach to detect the Toxoplasma genome in the ocular sample. In the second step, quantitative real-time PCR is used to measure the genomic DNA of T. gondii. By using this 2-step PCR method, it was possible to detect an exceedingly small amount of nucleic acid in small amounts of an ocular sample with a sensitivity of $85 \%$. 
Table 1. Recommended diagnostic tests based on immune status

\begin{tabular}{lllll}
\hline & Diagnostic test & $\begin{array}{l}\text { Immunocom- } \\
\text { petent patients }\end{array}$ & $\begin{array}{l}\text { Immunocom- } \\
\text { promised patients }\end{array}$ & Reference \\
\hline Indirect detection/serology & Serum IgG levels & + & $+{ }^{1}$ & $\begin{array}{l}\text { Montoya and Liesenfeld [10] } \\
\text { Montoya and Liesenfeld [10] } \\
\text { Rothova et al. [43] }\end{array}$ \\
& Serum IgM levels & + & - & Garweg et al. [51], Villard et al. [54] \\
\hline Girect detection & PCR & + & + & \\
\hline
\end{tabular}

Table 2. Treatment recommendations for toxoplasmosis

\begin{tabular}{lll}
\hline Condition & Treatment regimen & Reference \\
\hline Ocular toxoplasmosis & Pyrimethamine and sulfadiazine & Eyles and Coleman [59] \\
& Trimethoprim-sulfamethoxazole & Opremcak et al. [61] \\
& Butler et al. [57] & Soheilian et al. [68] \\
& IV clindamycin and dexamethasone & Bosch-Driessen et al. [69] \\
$\begin{array}{l}\text { Prophylaxis } \\
\begin{array}{l}\text { Pregnancy (6 months prior to conception } \\
\text { or up to 18 weeks) }\end{array}\end{array}$ & Pyrimethamine and azithromycin & Silveira et al. [66] \\
Pregnancy (18 weeks or later) & Trimethoprim-sulfamethoxazole & Montoya and Remington [46] \\
\hline
\end{tabular}

IV = Intravitreal. Corticosteroids may be added to the treatment regimen as recommended by Holland and Lewis [58].

\section{Treatment and Management}

In immunocompetent patients, Toxoplasma-related chorioretinitis is usually a self-limited infection and generally resolves spontaneously in a period of 4-8 weeks [57]. Considering the natural history of this disease and the side effects of medications, the risk of treatment secondary to the potential toxicity of antiparasitic medications may be greater than any benefit provided. However, treatment is recommended for lesions within the vascular arcades, adjacent to the optic disk, or larger than 2 optic disk diameters to reduce the chance of vision loss [58]. Toxoplasma retinochoroiditis in immunocompromised patients requires immediate treatment. Atypical presentations also warrant treatment [11]. Table 2 summarizes the current treatment recommendations for toxoplasmosis.

For more than 60 years, the combination of pyrimethamine and sulfadiazine, which have a synergistic effect on different steps of the nucleic acid synthesis in T. gondii, and corticosteroids have remained as the classic 'triple- drug therapy' [59]. Pyrimethamine inhibits dihydrofolic acid reductase, and sulfadiazine is a competitive antagonist of paminobenzoic acid. In a clinical trial by Rothova et al. [60], patients treated with the triple-drug therapy showed a greater reduction in the size of the retinal lesion compared with patients receiving other treatment regimens or no treatment. The authors concluded that tripledrug therapy was a good choice for patients with sightthreatening lesions.

In 1992, Opremcak et al. [61] studied trimethoprimsulfamethoxazole as a new treatment option for ocular toxoplasmosis. In this study, patients had resolution of active retinochoroiditis associated with improved vision. The authors concluded that trimethoprim-sulfamethoxazole was a safe and effective substitute for sulfadiazine and pyrimethamine in treating ocular toxoplasmosis. Trimethoprim-sulfamethoxazole is an inhibitor of folic acid metabolism and can cause bone marrow suppression. Therefore, baseline laboratory tests including a complete blood count, liver enzyme and serum creatinine levels, along with follow-up testing in 5-7 days, should be 
considered. If pancytopenia is identified, therapy should be discontinued and consultation with a hematologist should be considered. Moreover, sulfamethoxazole is the most widely used sulfonamide and use of this drug may cause sulfonamide hypersensitivity reactions. Sulfonamide hypersensitivity is classically characterized by fever, a generalized maculopapular rash, and toxicity of one or more internal organs, which may be asymptomatic [62]. This reaction usually develops 7-14 days after initiating therapy and resolves 7-14 days after the discontinuation of the drug [63]. Anaphylactic reaction is uncommon [64]. Sulfamethoxazole reactions may rarely result in toxic epidermal necrolysis and Stevens-Johnson syndrome, though they account for the largest percentage of the antibiotic-induced cases worldwide [65]. Trimethoprim-sulfamethoxazole contraindications include known hypersensitivity to trimethoprim and sulfonamides, pregnancy, severe hepatic and/or renal failure, and serious hematological disorders. Trimethoprim-sulfamethoxazole is contraindicated in pediatric patients under the age of 2 months.

In addition to the treatment of active infection, trimethoprim-sulfamethoxazole has a prophylactic role in preventing recurrent ocular toxoplasmosis $[66,67]$. In a study by Silveira et al. [66], 124 patients with a history of recurrent Toxoplasma chorioretinitis were randomized to an untreated control group or treatment group receiving trimethoprim-sulfamethoxazole ( $160-800 \mathrm{mg}$ ) every 3 days for 20 consecutive months. T. gondii chorioretinitis recurrence rates in control and treatment groups were 23.8 and 6.6\%, respectively [68]. Felix et al. [67] performed ocular toxoplasmosis prophylaxis by trimethoprim-sulfamethoxazole (160-800 mg) twice daily for 45 days. No recurrent toxoplasmosis chorioretinitis was detected in the treatment group while the recurrence rate was $12.8 \%$ in the placebo group within 12 months. Prophylaxis is recommended for patients with a history of severe or frequent recurrences. It may be considered for patients with vision-threatening lesions including those with retinochoroidal scars adjacent to the fovea, a critical anatomical location where any reactivation may increase the chance of severe vision loss (fig. 1) [67].

Clindamycin is a lincosamide antibiotic, which concentrates in ocular tissues and penetrates tissue cyst walls. Clindamycin is often added to the classic triple therapy as part of the 'quadruple therapy' [58]. Recent studies focused on the intravitreal injection of clindamycin and dexamethasone as a local treatment option. This method of drug administration delivers high concentrations of therapeutics into the vitreous cavity and retina, while re- ducing systemic adverse effects. In 2011, Soheilian et al. reported the results of their prospective randomized clinical trial aimed at comparing the efficacy of intravitreal injection of clindamycin and dexamethasone with the classic treatment for ocular toxoplasmosis [68]. The investigators assigned 68 patients to 2 treatment groups. The classic treatment group consisted of 34 patients, received pyrimethamine $25 \mathrm{mg}$ daily (initial dose of $75 \mathrm{mg}$ daily for 2 days), sulfadiazine $500 \mathrm{mg}$ every $6 \mathrm{~h}$ (initial dose of $4 \mathrm{~g}$ daily for 2 days), and $5 \mathrm{mg}$ folinic acid daily for 6 weeks, as well as oral prednisolone $1 \mathrm{mg} / \mathrm{kg}$ daily for 3 weeks starting from the third day of therapy. The intravitreal clindamycin plus dexamethasone group with 34 patients received an intravitreal injection of $1 \mathrm{mg}$ clindamycin plus $400 \mu \mathrm{g}$ dexamethasone with the mean number of injections of 1.6. Both treatment methods were successful in terms of lesion size reduction, visual acuity improvement, and vitreous inflammation reduction, and there were no statistically significant differences between the 2 groups. The authors concluded that intravitreal injection of clindamycin and dexamethasone might be an acceptable alternative to the classic triple-drug treatment in ocular toxoplasmosis. Advantages of intravitreal treatment include increased patient convenience, improved systemic side effect profile, greater drug availability, and fewer follow-up visits and hematological evaluations. Although intravitreal intervention appears to be an effective treatment option for immunocompetent patients, systemic therapy is recommended in immunocompromised patients to prevent toxoplasmosis-related complications in the fellow eye or elsewhere in the CNS.

A prospective randomized clinical trial compared the effects of two treatment regimens, pyrimethamine and azithromycin versus pyrimethamine and sulfadiazine, for the treatment of sight-threatening (near optic disk or fovea) ocular toxoplasmosis. The efficacy of the multidrug regimen with pyrimethamine and azithromycin was similar to the standard treatment with pyrimethamine and sulfadiazine. However, the frequency and severity of adverse effects were significantly lower in patients receiving pyrimethamine and azithromycin. This data supports multidrug therapy with the combination of pyrimethamine and azithromycin as an acceptable alternative for the treatment of sight-threatening ocular toxoplasmosis [69].

As in other ocular infections, the host immune response promotes intraocular inflammation against tachyzoites within the retina. The role of corticosteroids is to suppress the accompanying inflammation and minimize chorioretinal damage. The timing of initiation and 
the appropriate dose of corticosteroids are important to balance the suppression of the immune response to the parasite while minimizing the disease severity [70]. Corticosteroid therapy without antiparasitics may lead to large retinal lesions even in immunocompetent patients $[71,72]$. The baseline indications for the use of corticosteroids include severe vitreous inflammation, decreased vision, proximity of lesions to the fovea or optic disk and the large size of the active lesion [58]. The preferred drug for oral corticosteroid is prednisone at a dose of $0.5-1.0$ $\mathrm{mg} / \mathrm{kg} /$ day. The protocol for initiating corticosteroid therapy for patients with ocular toxoplasmosis varies greatly. In a survey by Holland and Lewis [58], 17\% of physicians reported using oral corticosteroid for all immunocompetent patients with ocular toxoplasmosis regardless of clinical findings. Corticosteroids were started simultaneously with antiparasitic drugs by $36 \%$ of respondents in this survey, while $64 \%$ deferred the start of corticosteroid therapy 1-7 days after starting antiparasitic therapy, with the majority waiting only 1-3 days for steroid initiation. Corticosteroid therapy is contraindicated in immunocompromised patients lacking the normal inflammatory response to the parasite [72]. Most ophthalmologists prefer topical steroids for ocular toxoplasmosis patients. In the survey by Holland and Lewis [58], 82\% of physicians reported giving topical steroids for immunocompetent patients with ocular toxoplasmosis. The main indications for use of topical corticosteroids are ocular pain, redness, photophobia, moderate to severe anterior chamber inflammation, and elevated intraocular pressure $[16,58]$.

The treatment of ocular toxoplasmosis during pregnancy requires special consideration due to the potential adverse effects of antiparasitic agents on the fetus [46]. If the mother acquires Toxoplasma infection during or immediately prior to pregnancy, there is a significant risk of placental transmission, and the risk increases with gestational age. Dunn et al. [73] reported an overall vertical transmission rate of $29 \%$. The risk of transmission in early pregnancy was low, $6 \%$ at 13 weeks of gestation, and increased greatly in the second and third trimesters of pregnancy reaching $72 \%$ at 36 weeks. Although the transmission rate is low, toxoplasmosis severity and morbidity are much higher in infants acquiring the infection in the early gestational period [73]. If a pregnant woman becomes infected up to 18 weeks into the pregnancy or within the 6 months prior to conception, treatment with the macrolide antibiotic spiramycin is recommended. Spiramycin does not readily cross the placenta, and there is no evidence for spiramycin teratogenicity, thus it is consid- ered not to have a teratogenic effect. Alternatively, local intervention with intravitreal clindamycin and dexamethasone could be considered to prevent the possible teratogenic effects of systemic pyrimethamine and sulfadiazine. If the maternal infection is acquired 18 weeks or later after conception, treatment with pyrimethamine, sulfadiazine and folinic acid is advised [46].

\section{Conclusions}

Ocular toxoplasmosis is the most common cause of infectious posterior uveitis, and the diagnosis is made by clinical findings in the majority of the cases. Laboratory confirmation relies on the analysis of serum or intraocular samples for antibody detection, but PCR is becoming more widely available for direct identification of the parasite DNA in the eye, and sensitivity of PCR is improving with new methods of detection. Although the classic triple-drug therapy of pyrimethamine, sulfadiazine, and corticosteroid is an effective choice, alternative treatment regimens including single agent treatment with trimethoprim-sulfamethoxazole, intravitreal injection of clindamycin with dexamethasone, or combination of azithromycin with pyrimethamine have been shown to be effective against ocular toxoplasmosis. The use of the trimethoprim-sulfamethoxazole combination is now preferred by many due to better patient compliance, faster resolution of chorioretinitis, and improved visual acuity. Trimethoprim-sulfamethoxazole is well tolerated for long-term prophylaxis in high-risk patients. The intravitreal injections of clindamycin and dexamethasone as local treatment option provide high concentrations of therapeutic agents in the vitreous cavity and retina while avoiding most systemic side effects. The oral regimen of pyrimethamine and azithromycin has been reported to have excellent efficacy against ocular toxoplasmosis with significantly lower side effects compared to pyrimethamine and sulfadiazine combination. New developments in the diagnosis and treatment improve our ability to prevent or significantly limit vision loss from ocular toxoplasmosis.

\section{Disclosure Statement}

None of the authors have any proprietary interests or conflicts of interest related to this submission.
Ozgonul/Besirli 


\section{References}

$>1$ Jabs DA: Ocular toxoplasmosis. Int Ophthal- 19 Jakob E, Reuland MS, Mackensen F, et al: mol Clin 1990;30:264-270.

$>2$ McCannel CA, Holland GN, Helm CJ, et al: Causes of uveitis in the general practice of ophthalmology. UCLA Community-Based Uveitis Study Group. Am J Ophthalmol 1996; 121:35-46.

$>3$ Balasundaram MB, Andavar R, Palaniswamy M, Venkatapathy N: Outbreak of acquired ocular toxoplasmosis involving 248 patients. Arch Ophthalmol 2010;128:28-32.

$\checkmark 4$ Perkins ES: Ocular toxoplasmosis. Br J Ophthalmol 1973;57:1-17.

$\checkmark 5$ Atmaca LS, Simsek T, Batioglu F: Clinical features and prognosis in ocular toxoplasmosis. Jpn J Ophthalmol 2004;48:386-391.

6 Black MW, Boothroyd JC: Lytic cycle of Toxoplasma gondii. Microbiol Mol Biol Rev 2000; 64:607-623.

7 Bosch-Driessen LE, Berendschot TT, Ongkosuwito JV, Rothova A: Ocular toxoplasmosis: clinical features and prognosis of 154 patients. Ophthalmology 2002;109:869-878.

$>8$ Kim SJ, Scott IU, Brown GC, et al: Interventions for toxoplasma retinochoroiditis: a report by the American Academy of Ophthalmology. Ophthalmology 2013;120:371-378.

$>9$ Harrell M, Carvounis PE: Current treatment of toxoplasma retinochoroiditis: an evidencebased review. J Ophthalmol 2014;2014: 273506.

$>10$ Montoya JG, Liesenfeld O: Toxoplasmosis. Lancet 2004;363:1965-1976.

11 Maenz M, Schluter D, Liesenfeld O, et al: Ocular toxoplasmosis past, present and new aspects of an old disease. Prog Retin Eye Res 2014;39:77-106.

$>12$ Dubey JP, Jones JL: Toxoplasma gondii infection in humans and animals in the United States. Int J Parasitol 2008;38:1257-1278.

$>13$ Robert-Gangneux F, Dardé ML: Epidemiology of and diagnostic strategies for toxoplasmosis. Clin Microbiol Rev 2012;25:264-296.

14 Pappas G, Roussos N, Falagas ME: Toxoplasmosis snapshots: global status of Toxoplasma gondii seroprevalence and implications for pregnancy and congenital toxoplasmosis. Int J Parasitol 2009;39:1385-1394.

$\checkmark 15$ Holland GN: Ocular toxoplasmosis: the influence of patient age. Mem Inst Oswaldo Cruz 2009;104:351-357.

16 Dodds EM, Holland GN, Stanford MR, et al: Intraocular inflammation associated with ocular toxoplasmosis: relationships at initial examination. Am J Ophthalmol 2008;146:856865. e2.

17 Nguyen AM, Seve P, Le Scanff J, et al: Clinical and etiological aspects of uveitis: a retrospective study of 121 patients referred to a tertiary centre of ophthalmology (in French). Rev Méd Interne 2011;32:9-16.

-18 Accorinti M, Bruscolini A, Pirraglia MP, et al: Toxoplasmic retinochoroiditis in an Italian referral center. Eur J Ophthalmol 2009;19: 824-830. Uveitis subtypes in a German interdisciplinary uveitis center - analysis of 1,916 patients J Rheumatol 2009;36:127-136. JR: Ocular toxoplasmosis. I. Parasitology, epidemiology and public health. Clin Exp Ophthalmol 2013;41:82-94.

21 Khairallah M, Yahia SB, Ladjimi A, et al: Pattern of uveitis in a referral centre in Tunisia, North Africa. Eye (Lond) 2007;21:33-39.

22 Smith JA, Mackensen F, Sen HN, et al: Epidemiology and course of disease in childhood uveitis. Ophthalmology 2009;116:1544-1551. e1.

23 De-la-Torre A, Gonzalez G, Diaz-Ramirez J, Gomez-Marin JE: Screening by ophthalmoscopy for Toxoplasma retinochoroiditis in Colombia. Am J Ophthalmol 2007;143:354-356.

24 Das D, Bhattacharjee $\mathrm{H}$, Bhattacharyya PK, et al: Pattern of uveitis in North East India: a tertiary eye care center study. Indian J Ophthalmol 2009;57:144-146.

25 Glasner PD, Silveira C, Kruszon-Moran D, et al: An unusually high prevalence of ocular toxoplasmosis in southern Brazil. Am J Ophthalmol 1992;114:136-144.

26 Guerina NG, Hsu HW, Meissner HC, et al: Neonatal serologic screening and early treatment for congenital Toxoplasma gondii infection. The New England Regional Toxoplasma Working Group. N Engl J Med 1994;330: 1858-1863.

27 Webster JP, Lamberton PH, Donnelly CA, Torrey EF: Parasites as causative agents of human affective disorders? The impact of antipsychotic, mood-stabilizer and anti-parasite medication on Toxoplasma gondii's ability to alter host behaviour. Proc Biol Sci 2006;273: 1023-1030.

28 Vasconcelos-Santos DV, Machado Azevedo DO, Campos WR, et al: Congenital toxoplasmosis in southeastern Brazil: results of early ophthalmologic examination of a large cohort of neonates. Ophthalmology 2009;116:21992205. e1.

29 Safadi MA, Berezin EN, Farhat CK, Carvalho ES: Clinical presentation and follow-up of children with congenital toxoplasmosis in Brazil. Braz J Infect Dis 2003;7:325-331.

>30 Berrebi A, Assouline C, Bessieres MH, et al: Long-term outcome of children with congenital toxoplasmosis. Am J Obstet Gynecol 2010;203:552. e1-6.

31 Kijlstra A, Jongert E: Control of the risk of human toxoplasmosis transmitted by meat. Int J Parasitol 2008;38:1359-1370.

32 Jones JL, Dubey JP: Waterborne toxoplasmosis - recent developments. Exp Parasitol 2010; 124:10-25.

-33 Bowie WR, King AS, Werker DH, et al: Outbreak of toxoplasmosis associated with municipal drinking water. The BC Toxoplasma Investigation Team. Lancet 1997;350:173177.
20 Furtado JM, Winthrop KL, Butler NJ, Smith
34 Dodds EM: Toxoplasmosis. Curr Opin Ophthalmol 2006; 17:557-561.

35 Mets MB, Holfels E, Boyer KM, et al: Eye manifestations of congenital toxoplasmosis. Am J Ophthalmol 1996;122:309-324.

36 Delair E, Latkany P, Noble AG, et al: Clinical manifestations of ocular toxoplasmosis. Ocul Immunol Inflamm 2011;19:91-102.

37 Park YH, Nam HW: Clinical features and treatment of ocular toxoplasmosis. Korean J Parasitol 2013;51:393-399.

38 Wakefield D, Cunningham ET Jr, Pavesio C, et al: Controversies in ocular toxoplasmosis. Ocul Immunol Inflamm 2011;19:2-9.

39 Alwassia AA, Cho H, Adhi M, et al: Sequential optical coherence tomography images of retinal necrosis in acute ocular toxoplasmosis. Retin Cases Brief Rep 2013;7:98-101.

40 Goldenberg D, Goldstein M, Loewenstein A, Habot-Wilner Z: Vitreal, retinal, and choroidal findings in active and scarred toxoplasmosis lesions: a prospective study by spectral-domain optical coherence tomography. Graefes Arch Clin Exp Ophthalmol 2013;251:20372045.

41 Oréfice JL, Costa RA, Scott IU, et al: Spectral optical coherence tomography findings in patients with ocular toxoplasmosis and active satellite lesions (MINAS Report 1). Acta Ophthalmol 2013;91:e41-e47.

42 Harper TW, Miller D, Schiffman JC, Davis JL: Polymerase chain reaction analysis of aqueous and vitreous specimens in the diagnosis of posterior segment infectious uveitis. Am J Ophthalmol 2009;147:140-147. e2.

43 Rothova A, de Boer JH, Ten Dam-van Loon $\mathrm{NH}$, et al: Usefulness of aqueous humor analysis for the diagnosis of posterior uveitis. Ophthalmology 2008;115:306-311.

44 Suzuki LA, Rocha RJ, Rossi CL: Evaluation of serological markers for the immunodiagnosis of acute acquired toxoplasmosis. J Med Microbiol 2001;50:62-70.

45 Marcolino PT, Silva DA, Leser PG, et al: Molecular markers in acute and chronic phases of human toxoplasmosis: determination of immunoglobulin $\mathrm{G}$ avidity by Western blotting. Clin Diagn Lab Immunol 2000;7:384389.

46 Montoya JG, Remington JS: Management of Toxoplasma gondii infection during pregnancy. Clin Infect Dis 2008;47:554-566.

-47 Montoya JG, Huffman HB, Remington JS: Evaluation of the immunoglobulin $\mathrm{G}$ avidity test for diagnosis of toxoplasmic lymphadenopathy. J Clin Microbiol 2004;42:46274631.

48 Goldmann H, Witmer R: Antibodies in the aqueous humor (in German). Ophthalmologica $1954 ; 127: 323-330$.

49 De Groot-Mijnes JD, Rothova A, Van Loon $\mathrm{AM}$, et al: Polymerase chain reaction and Goldmann-Witmer coefficient analysis are complimentary for the diagnosis of infectious uveitis. Am J Ophthalmol 2006;141:313-318. 
50 Fekkar A, Bodaghi B, Touafek F, et al: Comparison of immunoblotting, calculation of the Goldmann-Witmer coefficient, and real-time PCR using aqueous humor samples for diagnosis of ocular toxoplasmosis. J Clin Microbiol 2008;46:1965-1967.

-51 Garweg JG, de Groot-Mijnes JD, Montoya JG: Diagnostic approach to ocular toxoplasmosis. Ocul Immunol Inflamm 2011;19:255-261.

52 Montoya JG, Parmley S, Liesenfeld O, et al: Use of the polymerase chain reaction for diagnosis of ocular toxoplasmosis. Ophthalmology 1999;106:1554-1563.

53 Fardeau C, Romand S, Rao NA, et al: Diagnosis of toxoplasmic retinochoroiditis with atypical clinical features. Am J Ophthalmol 2002;134:196-203.

54 Villard O, Filisetti D, Roch-Deries F, et al: Comparison of enzyme-linked immunosorbent assay, immunoblotting, and PCR for diagnosis of toxoplasmic chorioretinitis. J Clin Microbiol 2003;41:3537-3541.

55 Steeples LR, Guiver M, Jones NP: Real-time PCR using the $529 \mathrm{bp}$ repeat element for the diagnosis of atypical ocular toxoplasmosis. $\mathrm{Br}$ J Ophthalmol 2016;100:200-203.

56 Sugita S, Ogawa M, Inoue S, et al: Diagnosis of ocular toxoplasmosis by two polymerase chain reaction (PCR) examinations: qualitative multiplex and quantitative real-time. Jpn J Ophthalmol 2011;55:495-501.

-57 Butler NJ, Furtado JM, Winthrop KL, Smith JR: Ocular toxoplasmosis. II. Clinical features, pathology and management. Clin Exp Ophthalmol 2013;41:95-108.
58 Holland GN, Lewis KG: An update on current practices in the management of ocular toxoplasmosis. Am J Ophthalmol 2002;134:102114.

59 Eyles DE, Coleman N: Antibiotics in the treatment of toxoplasmosis. Am J Trop Med Hyg 1953;2:64-69.

60 Rothova A, Meenken C, Buitenhuis HJ, et al: Therapy for ocular toxoplasmosis. Am J Ophthalmol 1993;115:517-523.

61 Opremcak EM, Scales DK, Sharpe MR: Trimethoprim-sulfamethoxazole therapy for ocular toxoplasmosis. Ophthalmology 1992;99: 920-925.

62 Slatore CG, Tilles SA: Sulfonamide hypersensitivity. Immunol Allergy Clin North Am 2004;24:477-490, vii.

63 Dibbern DA Jr, Montanaro A: Allergies to sulfonamide antibiotics and sulfur-containing drugs. Ann Allergy Asthma Immunol 2008;100:91-100; quiz 100-103, 111.

64 Cribb AE, Lee BL, Trepanier LA, Spielberg SP: Adverse reactions to sulphonamide and sulphonamide-trimethoprim antimicrobials: clinical syndromes and pathogenesis. Adverse Drug React Toxicol Rev 1996;15:9-50.

65 Wanat KA, Anadkat MJ, Klekotka PA: Seasonal variation of Stevens-Johnson syndrome and toxic epidermal necrolysis associated with trimethoprim-sulfamethoxazole. J Am Acad Dermatol 2009;60:589-594.

66 Silveira C, Belfort R Jr, Muccioli C, et al: The effect of long-term intermittent trimethoprim/sulfamethoxazole treatment on recurrences of toxoplasmic retinochoroiditis. Am J Ophthalmol 2002;134:41-46.
67 Felix JP, Lira RP, Zacchia RS, et al: Trimethoprim-sulfamethoxazole versus placebo to reduce the risk of recurrences of Toxoplasma gondii retinochoroiditis: randomized controlled clinical trial. Am J Ophthalmol 2014;157:762-766. e1.

68 Soheilian M, Ramezani A, Azimzadeh A, et al: Randomized trial of intravitreal clindamycin and dexamethasone versus pyrimethamine, sulfadiazine, and prednisolone in treatment of ocular toxoplasmosis. Ophthalmology 2011;118:134-141.

69 Bosch-Driessen LH, Verbraak FD, SuttorpSchulten MS, et al: A prospective, randomized trial of pyrimethamine and azithromycin vs pyrimethamine and sulfadiazine for the treatment of ocular toxoplasmosis. Am J Ophthalmol 2002;134:34-40.

70 Oray M, Ozdal PC, Cebeci Z, et al: Fulminant ocular toxoplasmosis: the hazards of corticosteroid monotherapy. Ocul Immunol Inflamm 2015, Epub ahead of print.

71 Garweg JG, Stanford MR: Therapy for ocular toxoplasmosis - the future. Ocul Immunol Inflamm 2013;21:300-305.

72 Holland GN, Engstrom RE Jr, Glasgow BJ, et al: Ocular toxoplasmosis in patients with the acquired immunodeficiency syndrome. Am J Ophthalmol 1988;106:653-667.

73 Dunn D, Wallon M, Peyron F, et al: Motherto-child transmission of toxoplasmosis: risk estimates for clinical counselling. Lancet 1999;353:1829-1833. 